\title{
Closing in on the link between apoptosis and autophagy
} Brian Leber ${ }^{1,2}$ and David W Andrews ${ }^{1 *}$

\author{
Addresses: ${ }^{1}$ Department of Biochemistry and Biomedical Sciences, McMaster University, 1200 Main Street West Hamilton, ON, L8N 3Z5, Canada; \\ ${ }^{2}$ Department of Medicine, McMaster University, 1200 Main Street West Hamilton, ON, L8N 3Z5, Canada \\ * Corresponding author: David W Andrews (andrewsd@mcmaster.ca) \\ FI000 Biology Reports 2010, 2:88 (doi:10.3410/B2-88) \\ This is an open-access article distributed under the terms of the Creative Commons Attribution-Non Commercial License \\ (http://creativecommons.org/licenses/by-nc/3.0/legalcode), which permits unrestricted use, distribution, and reproduction in any medium, \\ provided the original work is properly cited. You may not use this work for commercial purposes. \\ The electronic version of this article is the complete one and can be found at: http://fl000.com/reports/b/2/88
}

\begin{abstract}
While there is a clear connection between apoptosis and autophagy, the mechanisms that regulate the interaction have been difficult to identify. The initial clue to the link was the observation that $\mathrm{Bcl}-2$ was located at the endoplasmic reticulum (ER), where it could prevent some forms of apoptosis and also bind to the autophagy regulatory protein Beclin-I. However, both of these enigmatic observations have been united with the discovery of the nutrient-deprivation autophagy factor-I (NAF-I) protein. As an ER-localized protein that enhances the interaction of $\mathrm{Bcl}-2$ and Beclin-I and that also binds to the pro-apoptotic protein Bik, NAF-I is perfectly placed to be a central regulator of the switch between autophagy and apoptosis.
\end{abstract}

\section{Introduction and context}

Over the past several years, extensive work has been done to elucidate the process whereby members of the $\mathrm{Bcl}-2$ protein family at the mitochondrial outer membrane regulate the decision phase that commits cells to apoptosis. The current consensus is that the pro-apoptotic $\mathrm{Bcl}-2$ family members Bax (Bcl-2-associated X protein) and Bak (Bcl-2 homologous antagonist/killer) exist as inactive monomers. After exposure of the cell to an apoptotic stimulus, these proteins change their conformation in the mitochondrial outer membrane, form oligomers, and permeabilize the membrane, allowing the contents of the inter-membrane space to escape. The released proteins initiate the downstream caspase cascade that executes apoptosis. The two other subclasses of the $\mathrm{Bcl}-2$ family (the $\mathrm{BCl}-2$ homology region 3 (BH3)-only proteins and the antiapoptotic members) have distinct roles in this process: $\mathrm{BH}$-only proteins activate Bax, Bak, or both, whereas the anti-apoptotic members such as Bcl-2 (B-cell lymphoma 2), Bcl-XL (B-cell lymphoma-extra large), and Mcl-1 (induced myeloid leukemia cell differentiation protein) inhibit the BH3-only proteins as well as Bax and Bak.

However, all three subclasses of the Bcl-2 family are also found at the ER. The critical event that initiates apoptosis at the ER is more contentious than the action of the mitochondrial outer membrane. This is complicated by observations suggesting that $\mathrm{Bcl}-2$ family proteins regulate both caspase-dependent apoptosis and autophagy (an independent cell death/survival pathway initiated at the ER). The laboratory run by Gordon Shore at McGill University, Montreal, has been a leader in investigating the consequences of the Bcl-2 family localization to the ER. They have previously shown that an ER-specific BH3-only family member, Bik, can bind to Bcl-2 located at the ER and cause apoptosis [1]. They also identified a Bcl-2associated protein that links Bcl-2 to autophagy. Significantly, a homozygous mutation in this protein that disrupts interaction with $\mathrm{Bcl}-2$ was recently identified in three pedigrees with Wolfram syndrome, a severe neurodegenerative disorder [2].

\section{Major recent advances}

By using a form of Bcl-2 specifically targeted to the ER (Bcl2-cb5) [3], the nutrient-deprivation autophagy factor-1 (NAF-1) protein was demonstrated to be required for Bcl-2 to antagonize the autophagy regulatory protein Beclin-1 [4]. Crosslinking and subsequent mass spectrometry were used to identify proteins that both bound to $\mathrm{Bcl} 2-\mathrm{cb} 5$ and were displaced by Bik. The most 
abundant protein found in this search was a previously recognized small $(15 \mathrm{kD})$ type 1 transmembrane protein containing a cytosolic iron-sulfur binding cluster designated NAF-1 [4]. When analyzed by immunofluorescence, NAF-1 seems to be localized to the ER. The data obtained using a variety of approaches including binding to glutathione S-transferase fusion proteins and immunoprecipitation of transfected cells, as well as the original crosslinking experiments, make it highly likely that the interaction between NAF-1 and $\mathrm{Bcl}-2$ at the ER is direct, although this has yet to be formally proven. Importantly, decreasing NAF-1 expression by silencing RNA did not affect apoptosis initiated by Bik binding to Bcl-2. NAF-1 knockdown does enhance both starvation-induced autophagy as well as Bik-dependent autophagy, mediated by the displacement of Beclin-1 binding to Bcl-2. Beclin-1 is an authentic BH3-only binding protein [5] that, when released from Bcl-2, participates in a multi-component complex that initiates the formation of autophagosome formation at the ER $[6,7]$.

In co-immunoprecipitation assays, NAF-1 was also shown to be present in a complex containing $\mathrm{Bcl}-2$ and the inositol triphosphate receptor (IP3R). This interaction may be functionally relevant because the effect of $\mathrm{Bcl}-2$ binding to IP3R on intraluminal calcium stores, a mechanism elegantly demonstrated by Clark Distelhorst's group [8], is also inhibited by knocking down NAF-1 expression.

\section{Future directions}

These important findings shed light on the regulation of both apoptosis and autophagy at the ER by Bcl-2. Functionally, the interaction between Beclin-1 and Bcl-2 is enhanced by NAF-1, indeed, the data from Chang et al. [4] strongly suggest that NAF- 1 binds directly to $\mathrm{Bcl}-2$. It is unclear whether or not NAF-1 also binds directly to Beclin-1 and therefore acts as an adaptor protein for the Bcl-2-Beclin-1 interaction, or alternatively whether NAF-1 binding alters the conformation of the $\mathrm{Bcl}-2 \mathrm{BH} 3$ binding region such that it binds to Beclin-1 more tightly. There is already support for the theory that binding of BH3-only proteins to $\mathrm{Bcl}-2$ at the ER alters $\mathrm{Bcl}-2$ conformation and function [9]. If proven to be true, this would be the first instance where a non-BH3-only protein would be shown to have a similar effect to Bid (BH3 interacting domain death agonist) or Bim (Bcl-2-like protein 11). This would mean that NAF-1 would function as a specific allosteric regulator, as it does not seem to alter the binding of Bcl-2 to whichever BH3-only protein is displaced by Bik to initiate apoptosis.

Although not yet proposed in the literature, the presence of NAF-1 in Bcl-2-IP3R complexes has intriguing implications for both the composition of the Bcl-2 complex at the
ER and the potential involvement of NAF-1 in regulating autophagy via IP3R. The functional importance of this interaction is highlighted by the recent observation that baseline activity of IP3R is required to mediate low-level calcium release from the ER, where it is taken up by the mitochondrial calcium uniporter [10]. In this organelle, it is required to turn on pyruvate dehydrogenase activity, mediating a switch from glycolysis to the Krebs acid cycle thereby enhancing oxidative phosphorylation. When this process is interrupted at any of the steps between calcium release and ATP generation, energy deprivation is sensed and macro-autophagy is initiated in an mTOR (mammalian target of rapamycin)-independent fashion. Thus, by facilitating the binding of $\mathrm{Bcl}-2$ to both Beclin and IP3R, NAF-1 may mediate the control of two separate arms of autophagic cell death or survival.

Therefore, NAF-1 may act as a switch between autophagy and apoptosis at the ER. It is intriguing to note that the other mutated gene associated with Wolfram's syndrome also encodes a transmembrane ER protein that regulates calcium homeostasis, suggesting that this is an important pathway for neuronal survival [11]. Investigating how NAF-1 interaction with $\mathrm{Bcl}-2$ modulates binding of partners through different binding domains will be essential to understanding how this switch is regulated. It will also be important to determine whether all these proteins are present in the same complex or whether some interactions are mutually exclusive. Finally, it will be interesting to find out whether regulation is achieved by different complexes (if they exist) competing with each other for limiting factors.

\section{Abbreviations}

Bak, Bcl-2 homologous antagonist/killer; Bax, Bcl-2associated X protein; Bcl-2, B-cell lymphoma 2; BH3, $\mathrm{Bcl}-2$ homology region 3; ER, endoplasmic reticulum; IP3R, inositol triphosphate receptor; NAF-1, nutrientdeprivation autophagy factor-1.

\section{Competing interests}

The authors declare that they have no competing interests.

\section{Acknowledgments}

Work from our laboratories cited in this review was supported by a Canadian Institutes of Health Research (CIHR) grant FRN 12517 to DWA and BL, and a Tier I Canada Research Chair in Membrane Biogenesis to DWA.

\section{References}

I. Germain M, Mathai JP, Shore GC: BH-3-only BIK functions at the endoplasmic reticulum to stimulate cytochrome $c$ release from mitochondria. J Biol Chem 2002, 277:18053-60. 
2. Amr S, Heisey C, Zhang M, Xia XJ, Shows KH, Ajlouni K, Pandya A, Satin LS, El-Shanti H, Shiang R: A homozygous mutation in a novel zinc-finger protein, ERIS, is responsible for Wolfram syndrome 2. Am J Hum Gen 2007, 81:673-83.

3. Zhu W, Cowie A, Wasfy G, Penn LZ, Leber B and Andrews DW: Bcl-2 mutants with restricted subcellular location reveal spacially distinct pathways for apoptosis in different cell types. EMBO J 1996, I5:4130-4I.

4. Chang NC, Nguyen M, Germain M, Shore GC: Antagonism of Beclin I dependent autophagy by $\mathrm{Bcl}-2$ at the endoplasmic reticulum requires NAF-I. EMBO J 2010, 29:606-18.

5. Oberstein A, Jeffrey PD, Shi $Y$ : Crystal structure of the BclXL-Beclin I peptide complex: Beclin I is a novel BH3-only protein. J Biol Chem 2007, 282: |3|23-32.

FI000 Factor 6

Evaluated by Peter Colman 19 Apr 2007

6. Axe EL, Walker SA, Manifava M, Chandra P, Roderick HL, Habermann A, Griffiths G, Ktistakis NT: Autophagosome formation from membrane compartments enriched in phosphatidylinositol 3-phosphate and dynamically connected to the endoplasmic reticulum. J Cell Biol 2008, 182:685-70I.
7. He C, Levine B: The Beclin I interactome. Curr Opin Cell Biol 20IO, 22:140-9.

8. Rong YP, Aromolaran AS, Bultynck G, Zhong F, Li X, McColl K, Matsuyama S, Herlitze S, Roderick HL, Bootman MD, Mignery GA, Parys JB, De Smedt H, Distelhorst CW: Targeting Bcl-2-IP3 receptor interaction to reverse Bcl-2's inhibition of apoptotic calcium signals. Mol Cell 2008, 31:255-65.

9. Kim PK, Annis MG, Dlugosz PJ, Leber B, Andrews DW: During apoptosis bcl-2 changes membrane topology at both the endoplasmic reticulum and mitochondria. Mol Cell 2004, 14:523-9.

FI000 Factor 9

Evaluated by Richard Youle 02 Jun 2004, Atan Gross 22 Jun 2004

10. Cárdenas C, Miller RA, Smith I, Bui T, Molgó J, Müller M, Vais H, Cheung KH, Yang J, Parker I, Thompson CB, Birnbaum MJ, Hallows KR, Foskett JK: Essential regulation of cell bioenergetics by constitutive InsP3 receptor $\mathrm{Ca2}+$ transfer to mitochondria. Cell 2010, 142:270-83.

II. Osman AA, Saito M, Makepeace C, Permutt MA, Schlesinger P, Mueckler M: Wolframin expression induces novel ion channel activity in endoplasmic reticulum membranes and increases intracellular calcium. J Biol Chem 2003, 278:52755-62. 